\title{
NEUTROPHIL-TO-LYMPHOCYTE RATIO PREDICTS POST- STROKE PNEUMONIA IN ACUTE ISCHEMIC STROKE
}

\author{
GENS R ${ }^{1}$, DE VOS A ${ }^{2}$, DE KEYSER ${ }^{1}{ }^{1}$, DE RAEDT $\mathbf{S}^{1}$ \\ ${ }^{1}$ DEPARTMENT OF NEUROLOGY, UNIVERSITAIR ZIEKENHUIS BRUSSEL, CENTER FOR NEUROSCIENCES, VRIJE \\ UNIVERSITEIT BRUSSEL, BRUSSELS, BELGIUM \\ 2 DEPARMENT OF NEUROLOGY, SINT-MARIA HALLE, BELGIUM
}

\section{BACKGROUND}

Pneumonia is a common complication after acute ischemic stroke (AIS). It occurs in $1-33 \%$ of patients ${ }^{1}$ and has been associated with increased mortality and poor functional outcome $^{2}$. More recently, elevated Neutrophil-to-Lymphocyte ratio (NLR) has been linked to poor outcome after $\mathrm{AIS}^{3}$.

\section{OBJECTIVE}

Our goal was to investigate the predictive value of NLR and other demographic, clinical and laboratory measures for post-stroke pneumonia (PSP) in the first week after AIS.

\section{METHODS}

\section{Stroke Database \\ UZ Brussel \\ 4999 patients}

\section{Exclusion criteria}

- Hemorrhagic stroke

- Stroke mimics

- Unclear time of onset

- Routine blood sampling $>24 \mathrm{~h}$ after onset of symptoms

- Previous hematologic/ inflammatory/autoimmune disorder

- Infections preceding stroke

- Use of antibiotics $>24 \mathrm{~h}$ before admission

- Use of immunosuppressants
Electronic record system

- Demographics: age, gender

- Clinical info: history, medication, stroke severity (NIHSS), systolic blood pressure (BP), diastolic BP, mean arterial pressure, level of consciousness, dysphagia, dysarthria, tube feeding prior to pneumonia

- Laboratory value: absolute neutrophil count, absolute lymphocyte count, absolute platelet count and CRP on admission

\section{2 eligible patients}

Pneumonia modified CDC diagnostic criteria At least one of the former and one of the latter criteria fulfilled:

(1) Abnormal respiratory examination, pulmonary infiltrates on chest $x$-rays

(2) Productive cough with purulent sputum, microbiological cultures from lower respiratory tract or blood cultures, leukocytosis, elevation of CRP

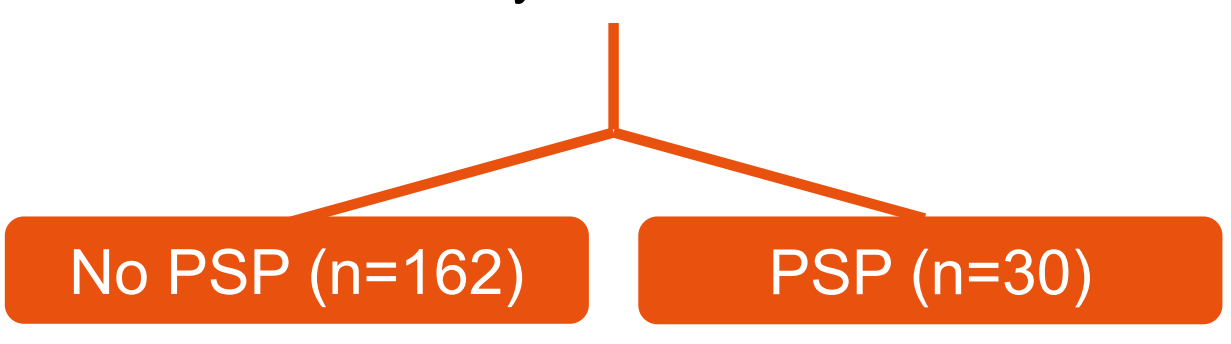

\begin{tabular}{|c|c|c|c|}
\hline \multicolumn{4}{|c|}{ RESULTS } \\
\hline \multicolumn{4}{|c|}{ Baseline characteristics of study population ( $n=192)^{*}$} \\
\hline Variables & Pneumonia $(n=30)$ & No pneumonia $(n=162)$ & p-value \\
\hline NIHSS & $17(11-21)$ & $7(3-14)$ & $<0.001$ \\
\hline NIHSS item 1.a & & & 0.001 \\
\hline Score 0 & $21(70.0)$ & $150(92.6)$ & \\
\hline Score 1 & $6(20.0)$ & $9(5.6)$ & \\
\hline Score 2 & $3(10.0)$ & $3(1.9)$ & \\
\hline NIHSS item 10 & & & $<0.001$ \\
\hline Score 0 & $3(10.0)$ & $65(40.6)$ & \\
\hline Score 1 & $8(26.7)$ & $70(40.3)$ & \\
\hline Score 2 & $19(63.3)$ & 25 (15.6) & \\
\hline Dysphagia & $25(83.3)$ & $58(35.8)$ & $<0.001$ \\
\hline Tube feeding & $18(60.0)$ & $23(14.2)$ & $<0.001$ \\
\hline ACE-Inhibitors & $13(43.3)$ & $37(22.8)$ & 0.019 \\
\hline Diuretics & $11(36.7)$ & $32(19.8)$ & 0.041 \\
\hline $\operatorname{ALC}\left(/ \mathrm{mm}^{3}\right)$ & $1516.2 \pm 606.2$ & $2175.1 \pm 974.6$ & $<0.001$ \\
\hline $\operatorname{ANC}\left(/ \mathrm{mm}^{3}\right)$ & $7719.6 \pm 2765.4$ & $5566.7 \pm 2410.8$ & $<0.001$ \\
\hline CRP & $7.0(1.8-13.1)$ & $3.0(1.3-6.5)$ & 0.020 \\
\hline NLR & $5.1(2.7-9.6)$ & $2.6(1.4-4.9)$ & $<0.001$ \\
\hline
\end{tabular}

*only significant results are shown

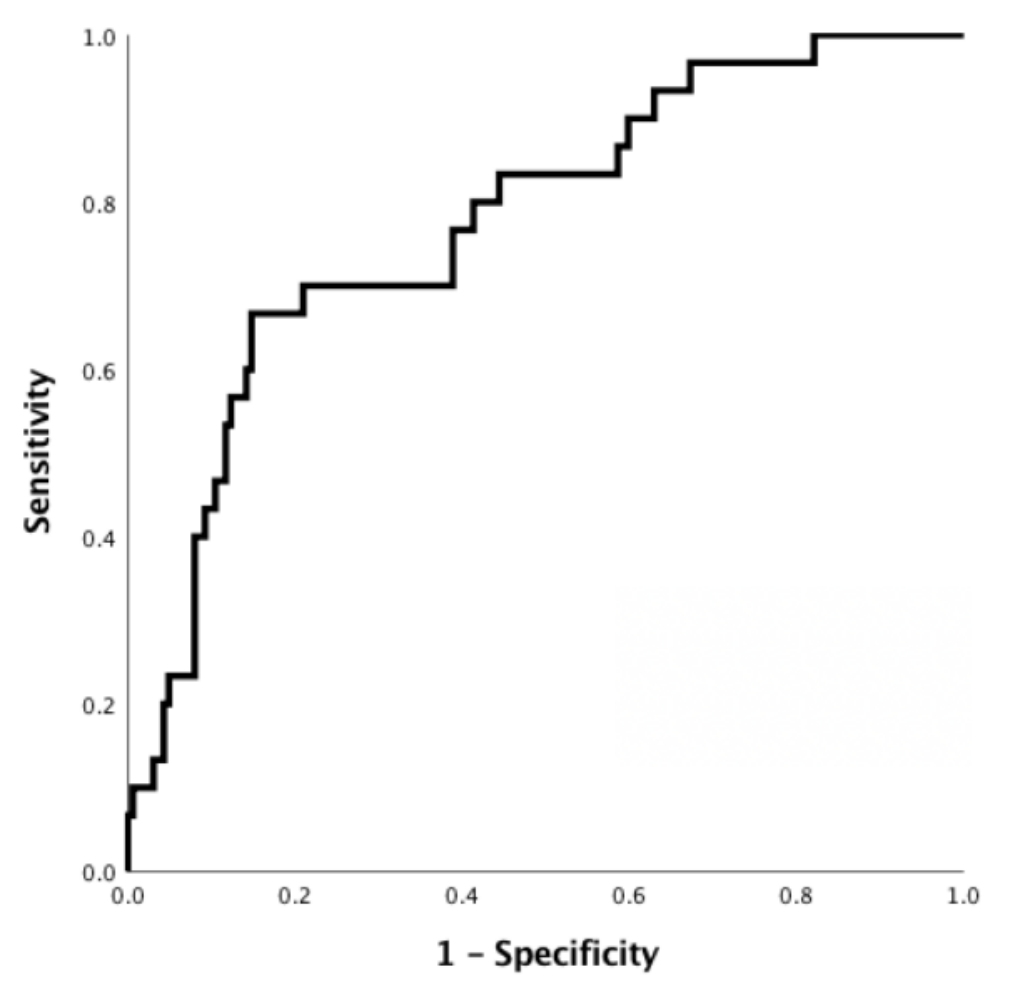

ROC curve analysis for NLR cut-off for NLR $=4.3$ with sensitivity $=70 \%$ and specificity $=79 \%$ $A U C=0.78(p<0.001)$

$\begin{array}{lccc}\text { Multiple logistic regression analysis } & & \\ \text { Variables } & \text { OR } & \mathbf{9 5 \% ~ C l} & \text { p-value } \\ \text { NIHSS item 10 score 1 } & 1.08 & 0.23-5.15 & 0.925 \\ \text { NIHSS item 10 score 2 } & 10.56 & 2.30-48.41 & 0.002 \\ \text { Dysphagia } & 6.31 & 1.90-20.95 & 0.003 \\ \text { NLR } \geq 4.3 & 11.88 & 3.83-36.88 & <0.001 \\ \text { Gender } & 5.06 & 1.55-16.47 & 0.007\end{array}$

\section{CONCLUSION}

Our results suggest that NLR, dysphagia, severe dysarthria and male gender are independent predictors of PSP in the first week after AIS.

\section{LITERATURE}

${ }^{1}$ Emsley et al. Acute ischaemic stroke and infection: recent and emerging concepts. Lancet Neurol 7, 341-353, doi:10.1016/S1474-4422(08)70061-9 (2008)

2 Aslanyan, S. et al. Pneumonia and urinary tract infection after acute ischaemic stroke: a tertiary analysis of the GAIN International trial. Eur J Neurol 11, 49-53 (2004)

${ }^{3}$ Fang et al. Higher neutrophil counts and neutrophil-to-lymphocyte ratio predict prognostic outcomes in patients after non-atrial fibrillation-caused ischemic stroke. Biomed J 40, 154-162, doi:10.1016/j.bj.2017.03.002 (2017). 